\title{
Knowledge based Recommendation Expert System for Diagnosis and Treatment of Diabetes
}

\author{
Minyechil Alehegn Tefera \\ Mizan Tepi University, Ethiopia
}

\begin{abstract}
Human life contacts with different problem when living in this world, including health problem. Those problems helps human being to find solutions for those problems using different mechanism, including technology. To tell honestly, there are so many healthcare is available, but they are not efficient. Now a day Artificial intelligence (AI) is one of the technology which helps human being and it has a vital role in the treatment and diagnosis of different diseases .from those crhonic and serious disease diabetes is the most difficult and easily untreatable disease especially in developing country like African country including Ethiopia and other in order to help the humans who lives with diabetes and reduce the death of human being by this disease we develop knowledge base which helps the patient by providing information how to identify the diseases and how to treat diabetes. The proposed system also reduces the wrong treatment. The proposed intelligent system aware the patient by providing information, descriptions, treatment and the type of the diabetes
\end{abstract}

\section{Keywords}

Diabetes, knowledge base, Artificial intelligence, Expert System, Treatment

\section{INTRODUCTION}

Now a day in this our global world different challenges are occurring. The challenges are not limited to those challenges are including health problem. As we now currently, Education, Healthcare industries, government and nongovernmental organization is worried about human life. Some health problems are very chronic and needs special treatment in order to save human life. Diabetes Diseases is clustered on such chronic diseases, therefore it needs special treatment in order to recover the patient. Mostly this diabetes disease (DD) found on women rather than men. In 2014, $8.5 \%$ of adults whose ages 18 and more than 18 had diabetes mellitus. By 2012 blood glucose was one of the causes of 2.2 million population death [1]. According to the International Diabetes Federation (IDF) report on 4 Nov 2017 more than 199 million women are living with diabetes as the report indicates .From those women most of them are reproductive based on the above information it is easy to think about this problem and it makes our heart think about it because those women are our mother, sister, wife and so on .As the report indicates in 2040 the number of women who are living with diabetes will increase from 199 million to 313 million [2].in this time this chronic and serious diseases killed a young people specially women throughout the world .In developing country like south Africa,sudan,nigeria,somalia,india,this Dangerous diseases distributed and killing up the young people that $\mathrm{t}$ affects both in economic and social development of the country Women are not aware how to treat the diseases using different mechanism including food type are recommended that is why we planned to do this system .this expert system has great role to minimize the death of people who have diabetes by providing advice and treatment for the patient as well as for the doctor. The new expert system can reduce the wrong treatment if the wrong treatment reduced it is easy to guess how much it is very advantages to minimize the number of people who are death by this chronic diabetes diseases .most of the time the peoples who are living with have not aware about the most common diabetes disease type and their symptoms as well as the treatment of each type of diabetes but such problem solved by this expert system the expert system not help only the patient it helps also for the doctor.

\section{RELATED WORK}

Million et al. [2] knowledge based expert system is one of the biggest branch of artificial intelligence (AI) and it is used to solve different complex and complicated problems. And also, it is not limited only computer science field it has a great role in various fields. Séverin et al. [1] Artificial intelligence has different application including in health industry by recommending the doctor as well as the patient.it is also used as a cognitive interaction with human beings. Samy et al. [3] Expert system has a great role now a day in health industry for recommendation and diagnosis purpose. SL5 programming language expert system was used in this review paper. The system was not complex for user. Samy and Rami [4]. Expert system (ES) is one of the main application of computer system. Es (Expert system) has different components such as inference engine, knowledge base, interface etc. Samy and Mohammed [5] develop an expert system that was helps as a recommendation for urination patient using SL5 Object expert system programming language.in this system yes or no question was used as a rule. Hsiao-Hsien et al. [6].in this study artificial neural network was used. And the system was online system. The selected diseases was diabetes mellitus and it was yes or no question answering. Samy and abed [7] in this study, they concentrated on the diagnosis of vomiting useful SL5 Object expert system object oriented programming language. it was very help full for the patient. Mainly it was concentrated providing information that used to aware about the disease and preventation mechanism.SL5 object expert programming language (OEPL) was used in this study.samy and Mohamed [8].mainly concentrated on diagnosis of shoulder problem using Clips object expert systems language (CLIP).it was very help full for the patent as well as for the parent. Yes or no question and answering was included in this system in order to solve shoulder problem. Ibrahim et al. [9] the study of diabetic system is very necessary in medical industry or health industry as well as diabetic patient in this study fuzzy rule was applied .this reasoning study helps for the doctor and patients. Different reasoning was applied including fuzzy rule. Form those reasoning the following were included $\mathrm{CBR}$ ( case based reasoning), OCR( ontology case based reasoning), FBR(fuzzy based reason ), and RBR(rule based reasoning) . From those different reasoning method CBR (case-based reasoning) was more effective, powerful, and less cost as in this study described. Kamadi et al. [10] an inference system (IS) can be discover $u$ by using knowledge discovery mechanism which 
used to retrieve unknown before. different decision rule was generated and developed.fals split was identified it concentrated on to minimize false split . Ibrahim et al. [11] an expert system is one of the computer programs which can provide decision, recommendation as well as advice similar to human being advice. This expert system (ES) was helpful for the doctor as well as the patient who have diabetes. any tool not used on this system. Tawfik et al. [12] VP_Expert Shell (VES) was used as an expert tool.an expert system has a great role in the area of all medical industries. knowledge Representation was applied as it was rule based expert system. . Baran and Hossein [13] ES(Expert system) is a power full to solve a difficult and complex problems.it is a program of computer which helps a human being to solve complex problems by feeding some programs. Chang-shing lee and Mei-Huj wang[14] was concentrated on two diseases Diabetes and Heart disease .fuzzy rule and semantic fuzzy decision making was applied. S.S .Abu Nasir et al.[15] AI(artificial intelligence) was defined as thinking about how to make the system act as the actual human being .expert system is applicable in different areas including health care industry as well as in agriculture . Orhan et al.[16] artificial neural network was applied on this study with model. It was concentrated on chest diseases. .Esin et al.[17] LDA-ANFIS was provide efficient accuracy.no recommendation system developed lack of awareness of information about the diabetic diseases has the chance to increase the disease and decrease the treatment. .kemal and salih p[18] Nero fuzzy inference system was applied. expert system also used in the prediction purpose in addition to recommendation and decision making.

\section{DIABETES}

Diabetes is the most series and very chronic diseases through the world without limited on only developing country it is also series in developed country. Diabetes mellitus defined as defect in the human body ability for the conversion of glucose to energy the cause of diabetes is when the human pancreas hormone which is known as insulin. treatment as it defined on the proposed recommendation system.

\subsection{Type 1 Diabetes}

It is one of the type of diabetes which is occurred by the production of less amount of insulin. This type of diabetes cover 5 to $10 \%$ it is also called insulin dependent diabetes.it has its own symptom and treatment as it defined on the result and the proposed expert system.

\subsection{Type 2 Diabetes}

This type of Diabetes also called as insulin independent diabetes diseases .this type of diabetes diseases is the most common and known disease in the world.it is caused by ineffective use of insulin in the world this type of diabetes covered from $80 \%$ to $90 \%$ in the world it has various treatment as it defined on the proposed recommendation system.

\subsection{Gestational Diabetes}

This type of diabetes occurred on pregnancies.in a total of only 2 to $4 \%$ covered in worldwide.

\section{EXPERT SYSTEM}

An expert system are can be defined as a computer programs which used to solve complex problems [12]. expert systems are used to simulate human being logic and reasoning. Expert systems are also known as knowledge base. In day to day life expert systems are have an important role to solve different problems in different area like health industry, transportation, financial industry, in education and other. Expert systems (ESs) are used in different companies like Google, IBM and another. In the proposed system this expert system applied on Health industry that is Diabetes diagnosis and treatment. Expert systems have the ability to perform as the same level of human being. And also, they are not easily stopped it indicates that expert systems have the behavior of reliability as human being .it is possible to produce more amount of expert system to increase the aveliability.it is not difficult to guess how much expert systems have an important role human being day to day activity including health treatment by providing. Expert systems are having different components. Those components are list as follow knowledge base, inference engine. These two components are main components of expert system.

\section{METHODS}

Based on the literature review that reviewed we plan to develop an expert system or recommendation system for diabetes diseases which helps to the patient as well as the doctor. There are different knowledge representation system from those the most common are fram, logic, semantic, and production rules. Knowledge in expert system has two parts Conventional programming and knowledge-based system in conventional programming

Programs $=$ Datastructure + Algorithm

In knowledge-based system the two key important words knowledge and inference provides expert system

ExpertSystem=Knowledge+inference 


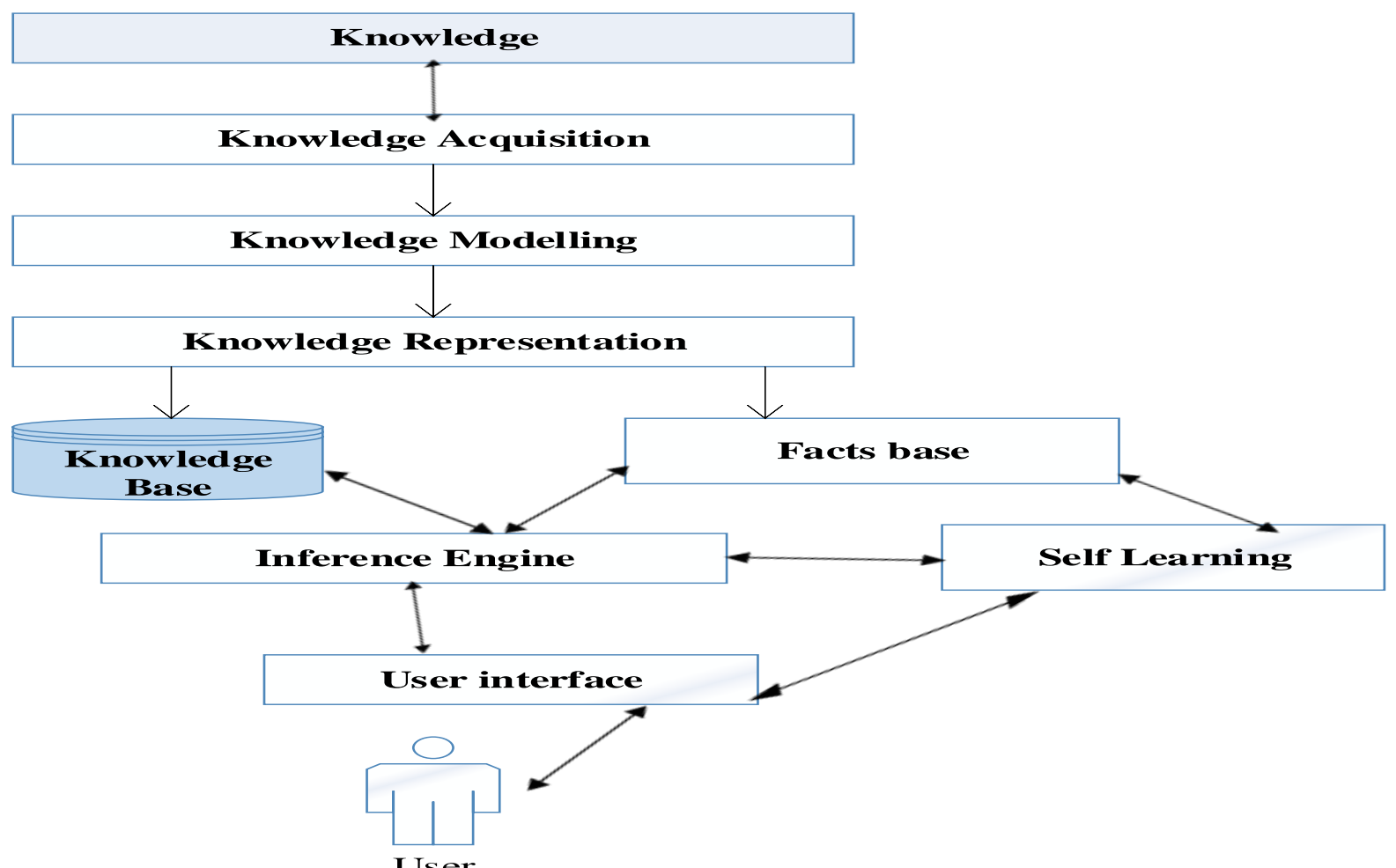

Fig. 1 Architecture of the proposed self-learning knowledge-based system (ASLKBS)

SWI-Prolog (AMD64, Multi-threaded, version 6.4.0)

File Edit Settings Run Debug Help

f:/sit III semi/weka dam/diagnosis1/m.pl.pl compiled 0.02 sec, 184 clauses 1 ? - start

MENU CHOICE

Type diabetes followed by full stop to get advice about Diabetes Diseases:

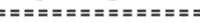

Please respond the questions by saying yes/no after choosing the diabetes

I: diabetes.

to proceed the advice?

does frequent urination ? (yes or no): yes.

is the vision change suddenly ? (yes or no): yes.

is increase appetite? (yes or no): yes.

is suddn weight loss? (yes or no): yes.

The Diabetes is type I.

Description

Type I diabetes the body does not produces

it is also called insulin dependant diabetes

people develope type 1 diabetes before their 40 th year.approximatly 5 to $10 \%$ all of diabetes cases are type I

Treatment

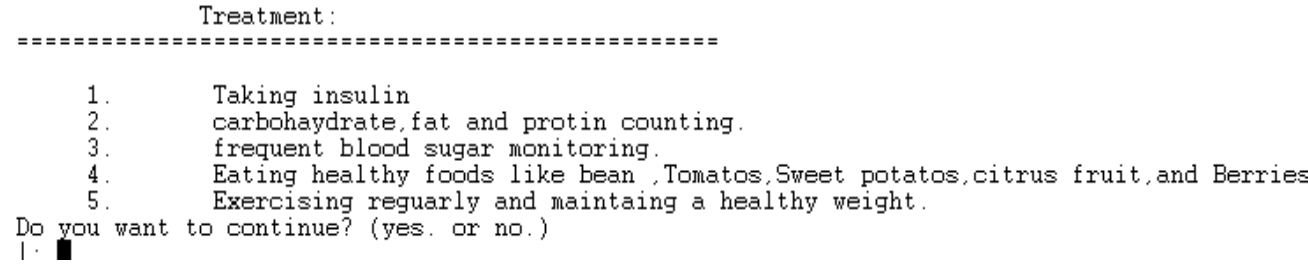

Fig .2 Diagnosis and treatment for Type I diabetes 
SWI-Prolog (AMD64, Multi-threaded, version 6.40)

File Edit Settings Run Debug Help

Do you want to continue? (yes. or no.)

|: yes.

does frequent urination? (yes or no): no.

is sexual problem? (yes or no): yes.

is there messel weakness ?(yes or no): yes.

is there tingling in your lower legs or feet?(yes or no): yes

is the blood pressure high? (yes or no): yes.

The Diabetes is type II.

Description:

the pancreas makes insuline, but it is either does not produce enough , or the insuline does not work properly

this typ occure nost of fen people who are over 40 years and overweight

$90 \%$ of people with diabetes have type II

Treatment:

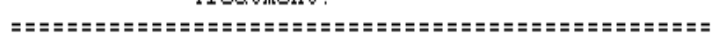

1. Exrcising

2. weight rlanagement

3. insulin injectiot

4. oral glucose lowering medication(taken by month)

Fig. 3 Diagnosis and treatment for Type II diabetes

SWI-Prolog (AMD64, Multi-threaded, version 6.4.0)

File Edit Settings Run Debug Help

Do you want to continue? (yes, or no.)

|: yes.

does frequent urination? (yes or no): no.

is sexual problem ? (yes or no): no.

is increasd thirst? (yes or no): yes.

is increased unrinary frequency ?(yes or no): yes.

is some times hungry ? (yes or no): yes.

The Diabetes is gestational diabetes.

Description

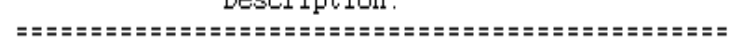

this type of diabees occures when their is a high bllood glucs level during pregnancy

As pregnancy progresses, the developing baby has a greater need for glucose

it occures in about $4 \%$ of all pregnancies

of feten ocures in women who have no prior history of diabetes

Treatment:

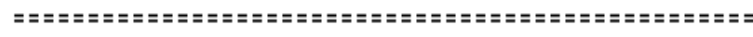

1. attention to maintaining aproper diet .

2. Regular Exercise

3. testing blood glucose levels to determine if your bwr write( lood glucose levels are at acceptable limitsl

4. asked to test your urine for ketones, substances that are produced when your body metabolizes fat

5 . not skiping meals

6 . institue insuline therapy.

Fig.4 diagnosis and treatment for Gestational diabetes 


\section{CONCLUSION}

The day is now to help people who live with diabetes, which is very serious and chronic disease in the world. The new prototype expert system helps to save human life at an early stage by providing advice, description, symptoms, and treatment for the patient. This expert system minimizes wrong treatment which is made by the doctor Especially in developing country like African countries, there is no enough doctors and laboratory technician to help the people who are Lives with diabetes, there for this expert system solve such problem. The most common types of diabetes disease mean that Type I, Type II, and Gestational Diabetes are included on this expert system with description, symptoms, and treatment of each type of diabetes. In future, it is recommended develop such like expert system for other diseases helps to save human life at initial stage.

\section{REFERENCES}

[1] Lemaignan, S., Warnier, M., Sisbot, E. A., Clodic, A., \& Alami, R. (2017). Artificial cognition for social humanrobot interaction: An implementation. Artificial Intelligence, 247, 45-69.

[2] Diriba, C., Meshesha, M., \& Tesfaye, D. (2016). Developing a Knowledge-Based System for Diagnosis and Treatment of Malaria. Journal of Information \& Knowledge Management, 15(04), 1650036.

[3] Naser, S. S. A., \& Mahdi, A. O. (2016). A proposed Expert System for Foot Diseases Diagnosis. American Journal of Innovative Research and Applied Sciences, 2(4), 155-168.

[4] Naser, S. S. A., \& AlDahdooh, R. M. (2016). Lower Back Pain Expert System Diagnosis and Treatment. Journal of Multidisciplinary Engineering Science Studies (JMESS), 2(4), 441-446.

[5] Naser, S. S. A., \& Shaath, M. Z. (2016). Expert system urination problems diagnosis. World Wide Journal of Multidisciplinary Research and Development, 2(5), 919.

[6] Rau, H. H., Hsu, C. Y., Lin, Y. A., Atique, S., Fuad, A., Wei, L. M., \& Hsu, M. H. (2016). Development of a web-based liver cancer prediction model for type II diabetes patients by using an artificial neural network. Computer methods and programs in biomedicine, 125, 58-65.

[7] Abu Naser, S. S., \& El-Najjar, A. E. A. (2016). An expert system for nausea and vomiting problems in infants and children.

[8] Naser, S. S. A., \& Hilles, M. M. (2016). An expert system for shoulder problems using CLIPS. World Wide
Journal of Multidisciplinary Research and Development, 2(5), 1-8.

[9] Ahmed, I. M., Alfonse, M., Aref, M., \& Salem, A. B. M. (2015). Reasoning Techniques for Diabetics Expert Systems. Procedia Computer Science, 65, 813-820.

[10] Varma, K. V., Rao, A. A., Lakshmi, T. S. M., \& Rao, P. N. (2014). A computational intelligence approach for a better diagnosis of diabetic patients. Computers \& Electrical Engineering, 40(5), 1758-1765.

[11] Ahmed, I., Mahmoud, A., Aref, M., \& Salem, A. (2013). A study on Expert Systems for Diabetic Diagnosis and Treatment. In Proceedings of International Conference on Recent Advances in Information Science (pp. 363367).

[12] Zeki, T. S., Malakooti, M. V., Ataeipoor, Y., \& Tabibi, S. T. (2012). An expert system for diabetes diagnosis. American Academic \& Scholarly Research Journal, 4(5), 1.

[13] Hashemi, B., \& Javidnia, H. (2012). An approach for recommendations in self management of diabetes based on expert system. International Journal of Computer Applications, 53(14).

[14] Lee, C. S., \& Wang, M. H. (2011). A fuzzy expert system for diabetes decision support application. IEEE Transactions on Systems, Man, and Cybernetics, Part B (Cybernetics), 41(1), 139-153.

[15] Abu-Naser, S. S., Kashkash, K. A., \& Fayyad, M. (2010). Developing an expert system for plant disease diagnosis. Journal of Artificial Intelligence, 3(4), 269276.

[16] Er, O., Yumusak, N., \& Temurtas, F. (2010). Chest diseases diagnosis using artificial neural networks. Expert Systems with Applications, 37(12), 7648-7655.

[17] Dogantekin, E., Dogantekin, A., Avci, D., \& Avci, L. (2010). An intelligent diagnosis system for diabetes on linear discriminant analysis and adaptive network based fuzzy inference system: LDA-ANFIS. Digital Signal Processing, 20(4), 1248-1255.

[18] Polat, K., \& Günes, S. (2007). An expert system approach based on principal component analysis and adaptive neuro-fuzzy inference system to diagnosis of diabetes disease. Digital Signal Processing, 17(4), 702710

[19] http://www.who.int/mediacentre/factsheets/fs312/en/ 1

[20] https://www.idf.org/our-activities/world-diabetesday/wdd-2017.html 